\title{
THE TUNING OF THE CAVITY OPTIONS FOR DIAMOND
}

\author{
C.L. Dawson, D.M. Dykes and P.A. McIntosh \\ CLRC Daresbury Laboratory, Warrington, WA4 4AD, UK.
}

\section{Abstract}

The RF group is considering both normally conducting [1] and super-conducting [2] cavity systems for DIAMOND. This paper will discuss using temperature and longitudinal deformation as methods of tuning for a normally conducting cavity. The longitudinal deformation of a super-conducting cavity is also discussed with its added technical difficulties of nanometer resolution and its cryogenic environment. Finite Element Analysis and URMEL-T [3] software has been used extensively to predict cavity geometry changes and the associated frequency shift.

\section{INTRODUCTION}

The current method of RF cavity frequency tuning on the SRS uses a non-contacting plunger. Recent experience at Daresbury has shown that normal plunger movement over a stored beam decay causes beam movement of $>20 \mu \mathrm{m}$ horizontally and $>10 \mu \mathrm{m}$ vertically. This movement is accommodated by the SRS, due to its relatively large source size, but must be avoided in DIAMOND.

Superconducting and warm systems are currently being considered, as a result tuning techniques for both systems are being assessed.

\section{NORMAL CONDUCTING RF}

At present, the normal conducting RF system for DIAMOND has been based on a frequency of $500 \mathrm{MHz}$. The storage ring RF system will consist of six $250 \mathrm{~kW}$ klystrons feeding six normal conducting cavities.

\subsection{The RF Cavity}

Following investigation and optimisation of the three most common geometry configurations (nose-cone, pillbox and bell-shaped) an optimised nose-cone cavity was selected for DIAMOND [4], shown in figure 1. The investigation of a tuning mechanism for a normal conducting system has been based on this geometry.

\subsection{Finite Element Analysis (FEA)}

FEA of the DIAMOND Cavity was performed using the Ansys ${ }^{\mathrm{TM}}$ Version 5.1 [5] Code. Cavity modelling is required to predict the dimensional changes that occur when the cavity temperature is changed, the force required to axially deform the cavity and to determine the stresses in the cavity, due to tuning techniques.

A 2-D axisymmetric model was constructed using Plane 55 thermal elements, which were switched to Plane 42 structural elements for structural analyses. The cavity will be manufactured and installed at room temperature, therefore a reference temperature of $20^{\circ} \mathrm{C}$ has been assumed for thermal strain calculations. The distribution of the RF power fluxes was calculated using URMEL-T and applied to the model. Each cavity will be required to dissipate $105 \mathrm{~kW}$ of RF power.

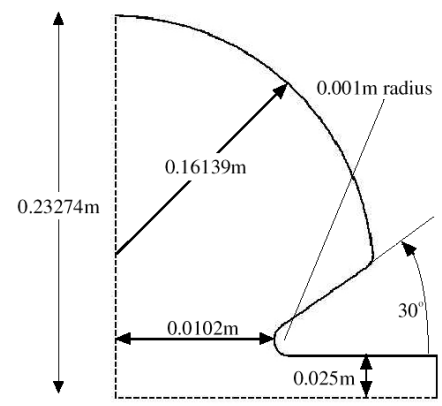

Figure 1. DIAMOND Cavity

\subsection{Tuning using Cooling Water Temperature Variation}

Operating the cavity at different temperatures changes the fundamental frequency of the cavity and the frequency of High Order Modes (HOM's). The temperature of the cavity can be changed by altering the temperature of its coolant. This technique has been used at many laboratories, including the SRS [6] and ELETTRA, to fight multibunch instabilities [7].

To confirm that the relationship between cavity geometry and temperature is linear, three analyses have been carried out. Analyses were carried out for bulk temperatures of $40^{\circ} \mathrm{C}, 50^{\circ} \mathrm{C}$ and $70^{\circ} \mathrm{C}$. A convection boundary condition was added to the model to simulate the water flow around the cavity with a film coefficient of $0.02 \mathrm{~W} / \mathrm{mm}^{2} /{ }^{\circ} \mathrm{C}$. A typical temperature contour result is shown in figure 2.

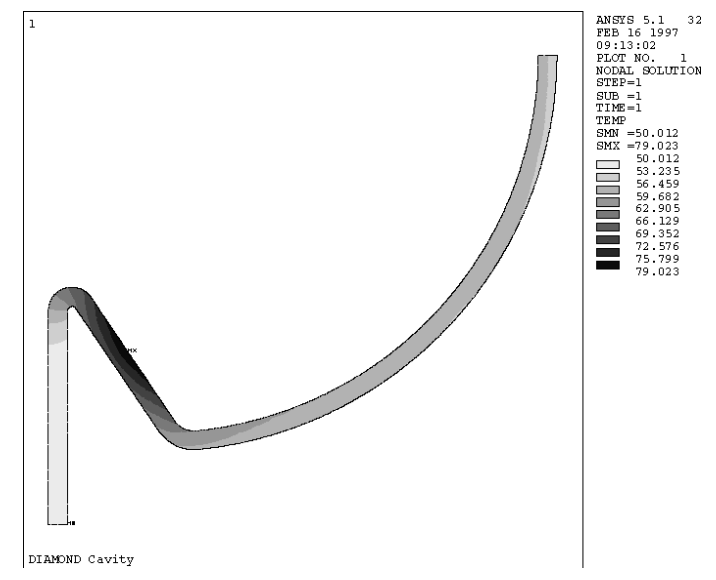

Figure 2. Temperature Contour Plot 
The area of maximum temperature rise is indicated on the diagram. In all three analyses the minimum cavity body temperature is the same as the temperature of the coolant and the maximum temperature is equal to the coolant temperature $+29^{\circ} \mathrm{C}$. This large deviation between the minimum and maximum temperatures can be reduced by careful design of the cooling channels.

Structural analyses were then carried out on each model to determine the cavity dimensional changes.

\begin{tabular}{||c|c|c||}
\hline $\begin{array}{c}\text { Water } \\
\text { Temp }\end{array}$ & $\begin{array}{c}\text { Max Cavity } \\
\text { Temp }\end{array}$ & $\begin{array}{c}\text { Movement per nose-cone into } \\
\text { the cavity }\end{array}$ \\
\hline $40^{\circ} \mathrm{C}$ & $69.023^{\circ} \mathrm{C}$ & $41.7 \mu \mathrm{m}$ \\
$50^{\circ} \mathrm{C}$ & $79.023^{\circ} \mathrm{C}$ & $69.9 \mu \mathrm{m}$ \\
$70^{\circ} \mathrm{C}$ & $99.023^{\circ} \mathrm{C}$ & $99.5 \mu \mathrm{m}$ \\
\hline
\end{tabular}

It is clear that this is a linear relationship, for each $1{ }^{\circ} \mathrm{C}$ change in cavity temperature there is $1.92 \mu \mathrm{m}$ nose cone movement. Figure 3 shows typical cavity deformation.

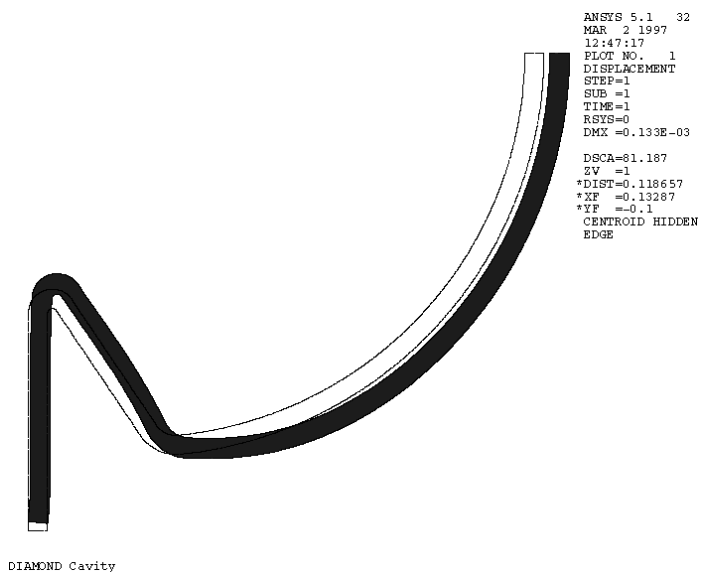

Figure 3. Deformed Cavity Shape

\subsection{URMEL-T Results}

This information was inputted into URMEL-T. Due to the geometry of the nose-cone cavity, the most significant frequency shift is due to the movement of the nose-cones along the axis of the cavity. For $1{ }^{\circ} \mathrm{C}$ rise in temperature the fundamental frequency shift of the cavity is $6.824 \mathrm{kHz}$. Using similar techniques to those used at other 3rd generation sources, cavity temperature can be controlled to $\pm 0.1^{\circ} \mathrm{C}$, giving a frequency resolution in the region of $682 \mathrm{~Hz}$.

\subsection{Tuning by Longitudinally Squeezing the Cavity}

If cavity temperature is used to prevent HOM instabilities, the fundamental frequency will also shift in addition to the troublesome HOM frequency. Therefore, a second tuning system is necessary to return the cavity to its fundamental frequency. The technique of squeezing the cavity on axis is being investigated. To prevent any waveguide sealing problems due to the squeezing of the cavity it is essential that the cavity is designed with all ports on the cavity centre-line. From URMEL-T calcula- tions, a $1 \mu \mathrm{m}$ movement of the nose-cones corresponds to a frequency shift of $3.554 \mathrm{kHz}$. The frequency range should be in the order of $200 \mathrm{kHz}$. A closed loop controls system with a frequency resolution of $500 \mathrm{~Hz}$ or better is required.

It is desirable to restrict the cavity movement so that it remains within the elastic region of the material to prevent any work-hardening of the cavity, ultimately leading to fatigue failure. This is achievable in the DIAMOND nose-cone cavity, as frequency shifts are relatively large for small nose-cone movements, but this produces a challenging mechanical resolution for the mechanism. A mechanical resolution of $0.14 \mu \mathrm{m}$ is required for a $500 \mathrm{~Hz}$ frequency resolution. If the option to select a $100 \mathrm{~Hz}$ frequency resolution is added, the mechanical resolution would need to be in the order of $28 \mathrm{~nm}$.

Using the 2-D model, it is anticipated that the force required to deform the cavity $\pm 40 \mu \mathrm{m}$ on axis will be in the region of $2 \mathrm{kN}$. The equivalent maximum stress in the cavity is $48.1 \mathrm{MN} / \mathrm{m}^{2}$, just below the $0.1 \%$ Proof Stress of Oxygen Free High Conductivity (OFHC) Copper, which is given in most texts to be $49-78 \mathrm{MN} / \mathrm{m}^{2}$. This will give a frequency range of $284.32 \mathrm{kHz}$.

Two methods of squeezing the cavity are currently being investigated, a hydraulic system and a left/right handed leadscrew system.

Hydraulic drives can produce large forces for the size of actuator and have an excellent power to weight ratio. Actuators are available with a $100 \mu \mathrm{m}$ range and a resolution of up to $1 \mathrm{~nm}$. However, the unit required to generate the hydraulic pressure is large and expensive. It is also difficult to prevent the vibration from the equipment being transmitted through the hydraulic fluid to the cavity [8].

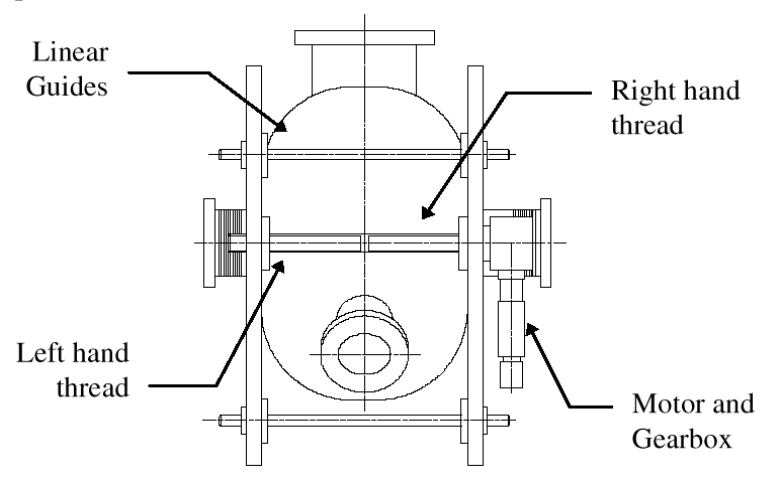

Figure 4. Leadscrew System

The left/right handed leadscrew, as shown in figure 4 , is a relatively low cost solution, but there are some disadvantages. The use of a leadscrew of any description means that the system will display backlash and thermal properties are poor. Most of these problems can be overcome by careful design and choice of leadscrew. A ball screw has very low backlash and high accuracy, but due to its low friction motor brakes would need to be fitted to prevent the cavity reactive force winding the ballscrew 
back. However, by deforming the cavity using frequency feedback, the accuracy of the leadscrew is less important and a conventional leadscrew could be used and the need for motor brakes eliminated. Thermal problems are not anticipated due to the environmental control of the DIAMOND tunnel. The main disadvantage with this system is the very high gear ratio required to obtain the specified resolution.

\section{SUPERCONDUCTING RF}

If superconducting technology is used, it is likely that DIAMOND will move to a $350 \mathrm{MHz}$ system, to take advantage of the development work of other sources in this area. Work has recently started on the super-conducting system and the development of the tuning system is much less advanced. This section will describe some preliminary thoughts on the subject.

\subsection{Additional Problems Associated with Tuning Super- conducting Cavities.}

There are more problems to be overcome when designing a tuning mechanism for a superconducting cavity. The cavity is housed in a helium cryostat and all components should be accessible for maintenance. Any mechanism used to modify the cavity geometry must make the transition from atmospheric to cryogenic temperatures. The geometry deformations required for tuning are in the nanometer region and speed of response is critical as the system must be capable of combating the frequency shifts and oscillations which can occur, due to changes in pressure in the cryostat.

\subsection{Methods of Super-conducting Cavity Tuning}

Two methods of tuning are usually required, a coarse tuner with a range in the order of $500 \mathrm{kHz}$ and a fine tuner working over approximately $10 \mathrm{kHz}$. There are several actuators available which are capable of nanometer resolution, three of the most common are discussed [9] here.

Piezoelectric materials experience a dimensional change on application of an electrical potential. Devices based on these materials are commercially available, with a range/resolution ratio of 10,000:1 and are capable of high response speeds. However, they are prone to hysteresis and creep, which limit the achievable accuracy. Actuators are available with a traverse of up to $100 \mathrm{~mm}$ in step sizes of $2 \mathrm{~nm}$ or less. However, in devices of this length, stiffness and response speed are compromised.

Electrostrictive devices are very similar to the piezoelectric actuator. However they exhibit reduced hys- teresis and have a low thermal expansion coefficient making them ideal for high accuracy applications. Simpler electrical drives may make them cheaper than the equivalent piezoelectric system. The main disadvantage is they are distinctly non-linear, but this is readily overcome with modern computer controls. In many cases the range is compromised to remain in a relatively linear portion of the working range. Hysteresis increases rapidly when operated below 273K, making any benefit over piezoelectric materials negligible.

Magnetostrictive devices are again very similar to piezoelectric actuators, but the strain is caused by the presence of a uniform magnetic field. The displacement per unit field is a linear relationship. They are ideal for heavy duty actuators working in the $100 \mu \mathrm{m}$ range. However, they are subject to hysteresis and creep.

\section{FUTURE WORK}

Work is progressing steadily on both systems, but there is a significant amount of work to do before comprehensive design schemes are available. The majority of the work on the warm system has been based upon the results taken from a 2-D axisymmetric model. The results must be confirmed on a complete 3-D cavity. Preliminary calculation work is to be started on the super-conducting cavity.

\section{ACKNOWLEDGMENTS}

The authors wish to thank all those who contributed to this paper. Particularly, M.J.Collier for his contributions to the design and A.R.Goulden for his advice on the cooling of RF cavities.

\section{REFERENCES}

[1] D.M.Dykes, 'The DIAMOND RF System', EPAC'96 Proceedings, p1943.

[2] D.M.Dykes, 'A Super-conducting RF System for DIAMOND', these Proceedings.

[3] U.Lauströer, URMEL+URMEL-T User Guide, DESY M-87-03, 1988.

[4] P.A.McIntosh, 'Comparison of RF Cavity Designs for 3rd Generation Light Sources', EPAC'96 Proceedings, p1955.

[5] Ansys Users Manual Rev.5, Swanson Analysis Systems Inc., DNR300:50, February 1994.

[6] P.A.McIntosh et al., 'Temperature dependant High Order Modes (HOM) in the SRS Cavities', EPAC'96 Proceedings, p1961.

[7] A.Fabris et al., 'Characterisation of the Low Level System of the ELETTRA RF Plants', EPAC'96 Proceedings, p1917.

[8] S.T. Smith and D.G. Chetwynd, 'Foundation of Ultra Precision Mechanism Design', Gordon and Breach Science Publishers, 1992.

[9] 'Proceedings of the $4^{\text {th }}$ Workshop on RF Superconductivity. Vol.1.', 14-18 August 1989, KEK, Japan. 